\section{ralud mental}

Flores Lázaro, Julio César; Saldaña García, Cinthya Nenetzyn; Ortega, José Marcos; Escotto Córdova, Eduardo Alejandro; Pelayo González, Héctor Juan Desarrollo del uso y la fluidez de verbos, su importancia para la neuropsicología Salud Mental, vol. 38, núm. 1, enero-febrero, 2015, pp. 59-65 Instituto Nacional de Psiquiatría Ramón de la Fuente Muñiz Distrito Federal, México
Salud Mental

ISSN: 0185-3325

perezrh@imp.edu.mx

Instituto Nacional de Psiquiatría Ramón

de la Fuente Muñiz

México

Disponible en: http://www.redalyc.org/articulo.oa?id=58238799009

- Cómo citar el artículo

- Número completo

- Más información del artículo

Página de la revista en redalyc.org

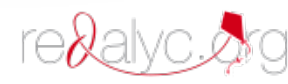

Sistema de Información Científica

Red de Revistas Científicas de América Latina, el Caribe, España y Portugal Proyecto académico sin fines de lucro, desarrollado bajo la iniciativa de acceso abierto 


\title{
Desarrollo del uso y la fluidez de verbos, su importancia para la neuropsicología
}

\author{
Julio César Flores Lázaro, ${ }^{1,2}$ Cinthya Nenetzyn Saldaña García, ${ }^{3}$ José Marcos Ortega, ${ }^{2,4,5}$ \\ Eduardo Alejandro Escotto Córdova, ${ }^{6}$ Héctor Juan Pelayo González ${ }^{7}$
}

Actualización por temas

\section{SUMMARY}

Syntactic competence development initiates during preschool age, an important component on this phenomena is active verb utilization, and morphology-semantic-verb acquisition. Recent approximations have outlined the importance of cognitive dependency-interaction during child development, for example the importance of executive control for verb selection (mapping) and efficient verb-utilization; as well as the importance of verb-semantics for cognitive processing; however at the national and the international context, developmental studies on verb-fluency and verb-morphology during verb-fluency paradigms are to date insufficient. In this article a basic review is presented on the characteristics of verb active utilization and verb-fluency in preschoolers and older children, outlining the utility for the field of developmental neuropsychology.

Key words: Verbal fluency, development, executive function.

\section{RESUMEN}

El desarrollo sintáctico del lenguaje se inicia entre los dos y tres años de edad. Dentro de este complejo proceso, destaca el uso activo del verbo y de sus variantes morfológicas-semánticas. En recientes investigaciones se ha resaltado la interdependencia de los procesos cognitivos durante el desarrollo, por ejemplo la probable dependencia del desarrollo del control ejecutivo para la selección (mapeo) y uso efectivo de los verbos, así como la importancia de los verbos en el procesamiento cognitivo. Sin embargo, tanto en el contexto nacional como internacional, los estudios sobre la fluidez de verbos y su organización léxico-semántica durante el desarrollo aún son insuficientes.

En este artículo se presenta una revisión básica sobre las características del desarrollo en el uso y la fluidez de verbos en niños preescolares y escolares. Se destaca su importancia para el campo lingǘstico y psicolingüístico, pero sobre todo para el campo de la neuropsicología del desarrollo.

Palabras clave: Fluidez verbal, desarrollo, función ejecutiva.

\section{INTRODUCCIÓN}

El desarrollo del lenguaje, y en particular la competencia lingüística básica en los niños, ocurre de una forma visiblemente acelerada en los primeros años de vida: alrededor de los tres años de edad los niños presentan una importante competencia lexical y gramatical. ${ }^{1}$ A pesar de esta competencia inicial, los niños se caracterizan por poseer sólo una fracción de la riqueza semántica sobre el significado y las relaciones léxico-semánticas y gramaticales de las palabras. Durante el enriquecimiento de las representaciones lingüísticas, con frecuencia los niños cometen errores de evocación por varias causas: no conocen la palabra de forma apropia- da, sus representaciones son incompletas o están en proceso de crear los mapas de las representaciones de palabras dentro del léxico. ${ }^{2}$

La capacidad predicativa y el uso eficiente de los verbos (morfología y semántica) requieren un periodo prolongado de desarrollo (hasta inicios de la adolescencia), aunque se ha descrito que desde alrededor de los 28 meses de edad los niños son competentes para determinar las relaciones sintácticas en las que ocurren los verbos (por ejemplo, verbos con dos argumentos: Pedro empujó a Lupita, o con un solo argumento: él duerme). De igual modo, ya pueden utilizar el orden de los elementos de la oración (la relación sujetoverbo-objeto) para extraer el rol del agente implicado en

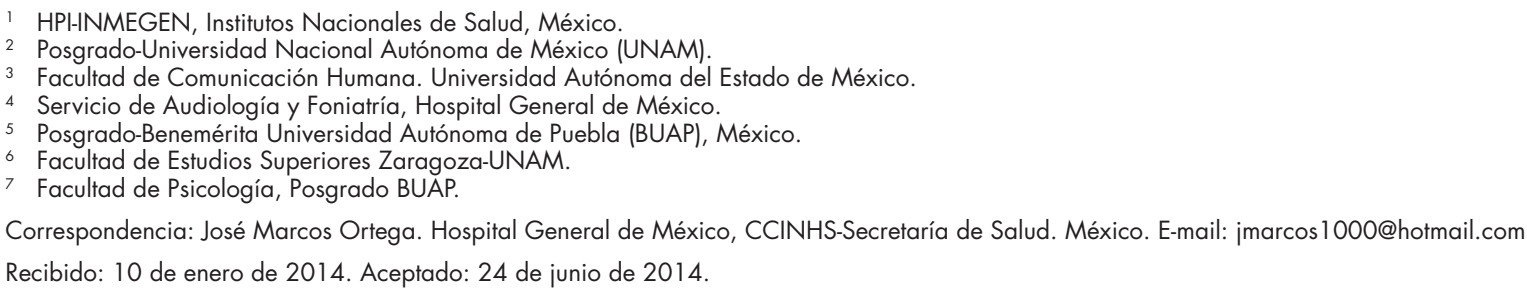


el verbo, como uno de los procedimientos principales de adquisición. ${ }^{3}$ Sin embargo, se ha observado que los niños de cuatro años de edad aún tienden a generar de forma espontánea innovaciones léxicas (cortillo para un instrumento que corta). Aun antes de utilizar este recurso, un número significativamente alto de niños tienden a emplear palabras ya existentes para indicar una acción o función. Se piensa que el uso de esta estrategia representa una solución cognitiva simple y más económica que generar una palabra nueva. Otro fenómeno que ocurre con frecuencia es recurrir a una descripción sintáctica (mediante una frase). Se cree que los niños perciben la necesidad de utilizar un verbo en el nuevo sustantivo o nombre del objeto que se les presenta y, debido a que su desarrollo morfológico aún no es suficientemente competente, se recurre al recurso sintáctico más fácilmente disponible. Con frecuencia, la forma previa a la esperada es una forma en infinitivo: primero cortar y, después, cortador. ${ }^{1}$

En la edad preescolar son comunes en el habla espontánea los verbos de alta frecuencia (correr, saltar, caminar), los cuales se convierten en los prototipos de categorías significativas mayores. ${ }^{4}$

\section{DENOMINACIÓN DE ACCIONES $Y$ EVENTOS EN LA ETAPA PREESCOLAR}

Hacia los tres años de edad se empieza a observar que los niños pueden relacionar de forma verbalmente competente un objeto con las acciones o funciones que realiza, y empiezan a aparecer los tiempos verbales. Es de particular interés el desarrollo desde la Holofrase: la expresión de todo un acto verbal a partir de una palabra. Se considera que el progresivo desarrollo cognitivo y lingüístico permitirá que el niño pueda analizar la secuencia de acontecimientos y asignar los recursos lingüísticos de forma específica y secuencial. ${ }^{5}$ De esta forma pasará de la frase nominal (uso del sujeto u objeto como verbo) y la frase predicativa, hacia la construcción de oraciones mínimamente estructuradas (marcando de forma específica cada sujeto u objeto).

El proceso de adquisición de verbos es más prolongado y complejo que el de sustantivos. ${ }^{6}$ Por ejemplo, a los cinco años de edad, aún los niños no han logrado la capacidad de expresar los verbos en infinitivo en forma consistente, utilizando con más frecuencia la forma en tercera persona. ${ }^{7} \mathrm{El}$ uso eficiente de los verbos es más complejo que el de otras categorías lingüísticas (por ejemplo, sustantivos) ya que si se aprende un verbo y su constelación de argumentos, no es posible generalizar dicho aprendizaje a otros verbos. ${ }^{8,9}$ En niños de tres a cinco años, el desempeño en tareas de denominación de verbos ha mostrado ser menos eficiente que el de sustantivos, lo cual es atribuido a las diferencias que subyacen en la representación y organización semántica de ambas categorías y a la forma distinta en la que son adquiridas. $^{8}$
Además de la organización semántica, es relevante explorar la competencia morfológica que subyace durante la niñez: la forma gramatical expresada (de los verbos que el niño conoce) en forma aislada o acompañada de argumentos. El paradigma estructural de un verbo en español puede resumirse en raíz, vocal temática, morfema I y morfema II; donde morfema I posee el tiempo, modo y aspecto; mientras que el morfema II representa la persona y el número. ${ }^{10}$ Dada la complejidad de la morfología verbal del español, se espera que de los seis a los 10 años aparezcan primero formas gramaticalmente sencillas acompañadas de algún argumento (María salta la cuerda). Al abstraerse en el modo infinitivo de los verbos la denominación de la acción, sería posible observar un predominio de verbos en infinitivo (saltar). ${ }^{9}$

Masterson, Drukes y Galiene ${ }^{8}$ estudiaron a niños de tres y cinco años de edad por medio de la Batería de Denominación de Objetos y Acciones, ${ }^{11}$ encontrando que el proceso de adquisición de verbos es posterior a la adquisición de sustantivos (mejor competencia de sustantivos). Hacia la edad de cinco años se empieza a observar un incremento significativo en la competencia de verbos; sin embargo, si además del número de aciertos se toman en cuenta los tiempos de reacción, aún se presentan importantes diferencias de desempeño a favor de los sustantivos. Los tiempos de reacción son interpretados como un proceso de búsqueda, con un importante efecto de selectividad-competencia entre las diversas opciones disponibles y/o la construcción de una alternativa.

En un estudio de denominación, utilizando láminas de figuras en blanco y negro (presentación por computadora) en niños de cinco y seis años, D'Amico, Devescovi y Bates ${ }^{7}$ encontraron que los niños de esta edad -en comparación con un grupo de adultos- presentan un 92\% de acuerdo ante la denominación lexical de objetos; sin embargo presentaron sólo un 52\% de acuerdo en la denominación de acciones. Los niños se caracterizaron por presentar denominaciones concretas (mendigar $v s$. pedir dinero), utilizar frases cortas en lugar de verbos simples como los adultos y por la tendencia a describir escenarios de contexto. Al comparar los ítems en donde se presentaron similitudes en la descripción, encontraron diferencias en los tiempos de reacción para denominar acciones (los niños presentaron mayor latencia), pero no encontraron diferencias significativas para denominar objetos. Los autores consideran que el uso de verbos requiere un espacio de decisión y de selección cognitivo (mapeo) más amplio y más complejo: diversas formas/alternativas para describir un mismo evento.

Los enfoques de dominios múltiples vs. los de dominio específico plantean una importante interacción entre diversos procesos cognitivos durante el desarrollo. ${ }^{12}$ De esta forma se plantea que el uso de tiempos verbales requiere también el desarrollo de una comprensión básica del tiempo y de los eventos que suceden temporalmente. Hacia los cuatro años, el uso de tiempos verbales mejora sustancialmente, aunque estos tiempos hacen referencia a sucesos inmediatos 
y a corto plazo. $^{2}$ En la actualidad, se hace énfasis en la interacción del desarrollo cognitivo en el manejo del tiempo, y una influencia recíproca en la adquisición del lenguaje; la capacidad para establecer relaciones predictivas se desarrolla de forma progresiva a lo largo de la infancia. ${ }^{13}$

\section{ORGANIZACIÓN SEMÁNTICA DE LOS VERBOS}

Algunos autores han planteado que los verbos conformarían una red semántica menos diferenciada que los sustantivos (en forma de matriz difusa), en donde el fenómeno de "intencionalidad" sería el nodo aglutinador principal para estos elementos. ${ }^{14}$ Se plantea que las fronteras entre las categorías de verbos que pertenecen a diferentes campos semánticos serían más difusas que las de los sustantivos, ${ }^{8}$ por lo que la generalización lexical y morfológica, así como el proceso de conformación de las redes semánticas para los verbos es más difícil de establecer que para otros elementos como los sustantivos, ya que los verbos difieren en el número y tipo de argumentos (roles de participación) que requieren: los intransitivos requieren sólo un argumento (el niño duerme), los transitivos requieren un sujeto y un objeto (Pedro pateó la pelota). Aun en la forma infinitiva se puede identificar el argumento de los verbos: un argumento, bostezar; dos argumentos, amar, patear; tres argumentos, poner, dar, comer.

Masterson et al. ${ }^{8}$ plantean también que los verbos no poseen un orden jerárquico entre sus categorías, el cual es característico de los sustantivos. La organización tendría una representación "plana" (sin jerarquías supra/subordinadas), una matriz difusa organizada con base en sus argumentos: presencia o ausencia de un instrumento ("martillar"), de un objeto ("colocar") de una dirección ("moverse a"), etc. Durante el desarrollo, los niños deben formar representaciones semánticas de los verbos y aprender las reglas que ligan dichas representaciones a las estructuras proposicionales. ${ }^{15}$

Las estructuras proposicionales pueden variar, pero no por ello varía la representación semántica del verbo; ésta se construye al escuchar la palabra en uso y ligarla con su concepto. Es correcto, por tanto, afirmar que los eventos se conceptualizan. ${ }^{15}$ En cuanto a su representación semántica, se ha propuesto que los verbos se podrían clasificar en dos tipos: verbos de movimiento, concretos (que incluyen un vasto set de asociaciones perceptuales y motoras) y verbos de cognición, abstractos (que incluyen una variedad de información proposicional y carecen del set de asociaciones perceptuales y motoras). A pesar de la relevancia que implica el verbo en el desarrollo del lenguaje expresivo, ${ }^{16}$ la organización semántica de los verbos es un campo inexplorado aún, por lo cual se desconocen las características de dicha organización en la niñez o en la edad adulta. ${ }^{8}$

Para el estudio de este campo se ha propuesto el modelo de cognición incorporada (Embodied Cognition Framework), el cual ya ha sido aplicado al proceso de la denominación de acciones. ${ }^{17}$ Este modelo plantea que la representación lexical de los verbos tiene dos modalidades: una motora (sensoriomotora) y otra semántica. Este enfoque permite estudiar si en su mayoría los verbos surgen de las representaciones sensorio-motoras y presentan una evolución hacia las semánticas (una transición concreto-abstracto). Recientes estudios de electrofisiología en adultos indican la coactivación de sistemas sensorio-motrices y semánticos durante la denominación y el procesamiento de acciones, con efectos de facilitación en el procesamiento cuando existe concordancia, y con efectos de competencia-bloqueo cuando existe incongruencia entre una acción y la oración que describe una acción. ${ }^{18}$ En el caso del desarrollo, se ha descrito que para los niños de dos años de edad es más fácil comprender oraciones que incluyen sujetos animados que inanimados. ${ }^{3}$

Thordardottir y Weismer ${ }^{19}$ encuentran en niños escolarizados de cinco a nueve años de edad, con desarrollo normal, un repertorio de ocho tipos de argumentos: agente, tema, objeto, fuente, meta, beneficiario, complemento copulativo, objeto+verbo+inespecífico, de los cuales, los que casi siempre aparecen en la expresión son fuente y objeto, siendo frecuente la omisión del sujeto si pragmáticamente es aceptable. Observan también en el desarrollo normal una variedad en el uso de tipos de argumentos y estructuras argumentales de hasta cuatro argumentos.

En tareas de denominación de verbos, el desempeño ha demostrado ser menos eficiente que el de sustantivos en edades entre tres y cinco años, lo cual es atribuido a las diferencias que subyacen en la representación semántica de ambas categorías y a la forma distinta en la que son adquiridas. ${ }^{8} \mathrm{La}$ organización y representación semántica de los verbos son más complejas que las de los sustantivos, pues deben incluir -debido a su estructura argumental- una relación semántica con sustantivos, además de un contexto sintáctico implícito que determine los argumentos que le pueden rodear. ${ }^{8}$

\section{FUNCIONES COGNITIVAS DEL VERBO}

Los verbos presentan un rol central gramatical: proveen un enlace entre el significado y la estructura de la oración, estableciendo las relaciones transitivas (acciones) entre los elementos de la oración. ${ }^{4}$ Desde el enfoque psicolingüísti$\mathrm{co}$, se plantea que los verbos codifican estructuras de argumentos que activan marcos de procesamiento sintácticos y semánticos, que van más allá de la denominación concreta de una acción. ${ }^{17}$ Se considera que los verbos actúan como unidades prototípicas para sintetizar categorías con mayor significado, lo que permite una simplificación sintáctica y semántica con un mínimo de pérdida de información. ${ }^{4}$ También se ha encontrado que incluso en niños preescolares los verbos ya conocidos les permiten inferir el significado de otras palabras. ${ }^{3}$ 


\section{NEUROPSICOLOGÍA DE LA FLUIDEZ DE VERBOS}

Aunque en contextos naturales, los verbos proceden y se desarrollan dentro del procesamiento sintáctico y pragmáti$\mathrm{co}^{8}$ algunos autores del campo de la cognición, pero sobre todo de la neuropsicología del desarrollo, plantean que la aproximación al verbo como una unidad de procesamiento cognitivo, y como una medida del funcionamiento neuropsicológico-ejecutivo, es de gran utilidad para evaluar el control ejecutivo-corteza prefrontal, y puede serlo también desde el contexto psicolingüístico., ${ }^{420,21}$ Además de los argumentos, otra característica a tomar en cuenta es una alta codependencia de las inflexiones, por lo que el contexto particular morfológico-sintáctico en donde se utilizan es un factor importante. Debido a esto, se plantea que la capacidad que subyace a la posibilidad de buscar, analizar y seleccionar la forma verbal adecuada tiene un alto componente de control ejecutivo. ${ }^{22}$

El interés por la organización cerebral de los verbos es muy reciente en comparación con el estudio sobre la organización cerebral del lenguaje. ${ }^{23}$ En todas las lenguas se reconoce a los verbos como categorías gramaticalmente distintas y, durante la adquisición, el aprendizaje de los verbos sigue un patrón diferente. ${ }^{8,24}$ Ante el daño cerebral, esta categoría se disocia claramente de los sustantivos en tareas de recuperación, denominación, lectura y escritura. ${ }^{25-27}$ Los verbos presentan una organización cerebral distinta a los sustantivos. Estudios muy recientes (con neuroimagen funcional) han encontrado que los procesos perceptuales de las acciones (ver a alguien correr) y el correlato semántico (qué significa "correr") se encuentran representados por redes de distintas regiones del cerebro. También se ha encontrado que la percepción de acciones activa de forma bilateral las regiones premotoras, pero la comprensión de oraciones que implican acciones físicas sólo activa las regiones premotoras izquierdas. ${ }^{28}$

En el marco del modelo Embodied Cognition, se plantea que el conocimiento conceptual no es amodal; se desarrolla en sistemas sensoriomotores que participan durante el procesamiento conceptual. Estos sistemas de patrones son reactivados durante la percepción y la acción. Con base en este modelo se han encontrado correlatos cerebrales distintivos para cinco tipos de información verbal: acción (corteza primaria y premotora), movimiento (corteza temporal-occipital), contacto (surco intraparietal y lóbulo parietal inferior), cambio de estado (corteza temporal ventral) y herramientas de uso (regiones temporales, parietales y frontales). ${ }^{29}$

Holland et al. ${ }^{20}$ estudiaron con neuroimagen funcional la fluidez de verbos en 33 sujetos desde siete a 18 años, encontrando activación (bilateral) en las áreas de Broca y de la región homóloga del hemisferio derecho, pero con tendencia a la lateralización al hemisferio izquierdo desde la niñez. También descubrieron que a medida que aumentaba la edad, esta lateralización se incrementaba aún más hacia el hemisferio izquierdo y se focalizaba particularmente a las porciones pos- teriores del giro frontal inferior (área de Broca). Encontraron igualmente que el número de puntos de activación (píxeles activos) en la CPF derecha disminuía con la edad. Resultados similares fueron encontrados por Wood et al. ${ }^{30}$ en 48 niños y 17 adultos. Szaflarski, Hoolland, Schmithorst y Byars ${ }^{31}$ encontraron que incluso desde los cinco años de edad se encuentra esta misma relación región cerebral-fluidez verbal.

La progresiva lateralización izquierda se relacionaría con los procesos de adquisición-complejización (influidos principalmente por las experiencias académicas y ambientales). Se ha planteado que la participación frontal izquierda implicaría la activación de representaciones abstractas (y no sensorioperceptuales) de las acciones. ${ }^{28}$ Por medio del desarrollo cognitivo, las representaciones lingüísticas requieren redes cerebrales más "abstractas" y semánticamente organizadas (de la corteza prefrontal, principalmente izquierda), por lo que hacia finales de la adolescencia, y sobre todo en sujetos adultos, los correlatos cerebrales son distintos que en los niños. ${ }^{32}$

\section{Control ejecutivo en la fluidez verbal}

La recuperación de verbos no sólo involucra un procesamiento morfológico y gramatical, involucra también una importante participación de funcionamiento ejecutivo. En tareas de fluidez de verbos, se ha encontrado que las opciones para elegir-seleccionar verbos involucran un componente ejecutivo y atencional mayor que el de los otros paradigmas pertenecientes a la categoría de fluidez verbal -fonológicos y semánticos-.$^{30}$ Por tanto, se ha considerado una medida de evaluación clínica del daño frontal durante la evaluación del funcionamiento ejecutivo. 33,34

Se considera que cuando un verbo es procesado, se coactiva en forma simultánea toda la variedad de argumentos que éste posee. ${ }^{35-37}$ Entonces, un verbo con múltiples argumentos, tomará más tiempo en ser procesado que un verbo con un solo argumento,; ${ }^{19}$ es decir, a mayor riqueza argumentativa en un verbo, mayores opciones (coactivaciones) para analizar y seleccionar (selectividad en la recuperación). Esto conduce a un mayor costo de procesamiento (mayor uso y por mayor tiempo de recursos cognitivos); por ende, mayor tiempo para desarrollar las tareas. El costo ejecutivo en el procesamiento de un verbo incluiría precisión en la búsqueda, uso de estrategias, actualización de la información y la producción de elementos en un tiempo dado. Todos estos aspectos están vinculados a la corteza prefrontal ${ }^{38}$ y específicamente a la región premotora y al área de Broca. ${ }^{21,39}$

Durante la ejecución de los paradigmas de fluidez verbal, y en particular cuando se deben realizar cambios entre las categorías de representaciones que están en competencia para su recuperación, hay un mecanismo funcional denominado "cambio" (switching) que subyace en la tarea y que se asocia con la actividad en el giro frontal inferior izquierdo. ${ }^{40}$ En niños hispanohablantes se ha observado en áreas de fluidez verbal una relación significativa entre el control inhibi- 
torio y la formación de racimos de palabras semánticamente relacionadas o clusters. La interpretación que se ha dado a esta relación es que en una evocación considerada como cognitivamente irregular por presentar lapsos de producción con lapsos de silencio, la intervención de las funciones ejecutivas permite la formación de clusters. ${ }^{41}$ Para el caso de la fluidez verbal, las varianzas en el desempeño se correlacionan directamente con el funcionamiento ejecutivo. ${ }^{42}$

\section{Eficiencia de uso y desarrollo ejecutivo (control ejecutivo)}

En el campo de las funciones ejecutivas se ha demostrado que los niños poseen conocimiento y habilidades cognitivas que con mucha frecuencia no utilizan de forma espontánea o voluntaria, ya que la capacidad para reclutar y seleccionar los mejores recursos cognitivos (en este caso argumentativos) requiere un desarrollo más prolongado. A este fenómeno se le denomina disociación saber-saber/hacer. ${ }^{13,43}$ En la medida en que su competencia ejecutiva (mapeo y selección) mejora, los niños presentan mejor capacidad para aprovechar de la forma más óptima su repertorio cognitivo; esta capacidad dependería principalmente del desarrollo de funciones ejecutivas. ${ }^{44}$

\section{Desarrollo concreto-abstracto}

El número de verbos producidos en un minuto se incrementa de forma lineal durante el desarrollo y no deja de aumentar hasta la juventud -en jóvenes que asisten a la universidad. . $^{33,45}$ Sin embargo, el tipo de emisión que se produce también cambia con la edad. Ávila ${ }^{46}$ encontró que cuando los niños mexicanos de tercer grado de primaria describen sus actividades por escrito utilizan verbos con referentes concretos en el $90 \%$ de los casos, en cambio, casi el 60\% de los niños de sexto grado de primaria utilizan verbos de tipo abstracto. De forma coincidente, se ha encontrado un progreso muy similar (concretoabstracto) en niños mexicanos en tareas de categorización semántica ${ }^{47}$ Estos resultados indican que durante el desarrollo, además de un incremento estadísticamente significativo en la productividad (número de verbos emitidos), lo cual reflejaría una medida de control ejecutivo (selectividad en la evocación-actualización de las representaciones), se esperaría un cambio cognitivo-cualitativo en el tipo de verbo (concreto vs. abstracto) y en la riqueza morfológica-semántica de los verbos producidos, la cual reflejaría una medida más cognitiva-psicolingüística y, sobre todo, más sensible clínicamente.

\section{Aplicación clínica}

En el campo de la neuropsicología se ha determinado que la denominación de objetos implica un proceso neurocognitivo menos complejo que la denominación de acciones; esto es debido a que el acceso a la etiqueta léxica de un objeto tiene una relación más directa y menor competencia semántica que la selección de la forma verbal adecuada.,14,22 Se ha encontrado que los niños con trastorno específico de lenguaje presentan mayores dificultades para denominar acciones que objetos, ${ }^{8,48}$ para determinar correctamente la inflexión verbal, para el uso de partículas, de la complementación, para el aprendizaje incidental de nuevos verbos, y para utilizar verbos con complejidad argumentativa -verbos con tres o más argumentos. ${ }^{20}$ Asimismo, diversos estudios con pacientes adultos con afasia de Broca han mostrado que la producción de verbos se dificulta en relación con el número de argumentos que requiere cada verbo. ${ }^{49}$

Baron, Erickson, Ahronovich, Baker y Litman ${ }^{50}$ encontraron que en niños de tres años de edad nacidos con bajo peso y pretérmino (<26 semanas de gestación), el desempeño de la fluidez verbal se ubicó por debajo del grupo control (nacidos a término y con un peso "normal"); en otros estudios con grupos similares se ha encontrado replicación de estos resultados, por lo que se ha sugerido que la fluidez de verbos puede ser un predictor temprano de disfunción ejecutiva/dificultades de fluidez de lenguaje durante la infancia. ${ }^{50}$

En niños que presentan traumatismo craneoencefálico también se ha utilizado la fluencia de verbos como parte de los instrumentos neuropsicológicos de evaluación, ${ }^{51}$ observándose un desempeño significativamente inferior con respecto al del grupo control. En el caso de niños que presentan epilepsia parcial simple, la producción de verbos se ve afectada. Midiendo la fluidez de tres tipos de verbos (auxiliares, copulativos y no infinitivos) y comparando el desempeño con un grupo control, la producción de verbos auxiliares se vio limitada correspondiendo con un estilo telegráfico. ${ }^{52}$

En niños con distrofia muscular se observó una narración cualitativamente inferior al grupo control con desarrollo normal, consistente en un número menor de verbos y de oraciones completas. Lo anterior sugiere que la reducida producción de oraciones se debe a un problema selectivo en la generación de la estructura argumental del verbo. ${ }^{53}$ Las diferencias en la narración también se han observado en población de niños que presentan dificultades en el desarrollo del lenguaje, ya que en la conversación aparecen formas verbales más complejas que en tareas de narración. ${ }^{54}$

Con respecto a los niños con trastorno del lenguaje, se ha encontrado que presentan retraso o modificación de uso en formas verbales, ${ }^{16}$ un mayor uso de verbos con significado no específico (hacer, estar) ${ }^{54}$ una menor variedad en el tipo de argumentos que acompañan al verbo y en el número de argumentos que se estructuran en la oración, ${ }^{19}$ sustitución de tiempos verbales, persona, menor variedad en el uso del aspecto (modo) verbal, ${ }^{55-57}$ latencias en tareas de denominación de verbos, ${ }^{8,48}$ así como una menor precisión en la denominación de verbos (acciones) con respecto a los sustantivos (objetos). ${ }^{48}$

Al realizar la comparación de dos casos con daño prefrontal-dorsolateral focalizado en dos adolescentes de 12 
años, uno con daño derecho y otro con daño izquierdo, Pérez Morales, Bittencourt Chastinet y Flores Lázaro ${ }^{58}$ describen que para el caso con daño prefrontal izquierdo se pierde la posibilidad de actualizar el verbo en forma infinitiva. Es decir, extrayéndolo de sus argumentos, por lo que la tarea se realiza con presencia de frases. En contraste, el caso con daño prefrontal derecho muestra un desempeño marginal para la generación de verbos en forma infinitiva. Lo anterior indica que ya a principios de la adolescencia se podrían encontrar diferencias de lateralización, con una mayor contribución de la corteza prefrontal izquierda para la generación de verbos como unidades lingüísticas individuales.

Las evidencias provenientes de la neuroimagen funcional, sumadas a las evidencias clínicas, permiten plantear que la fluidez de verbos puede ser una medida más sensible a la disfunción frontal, ${ }^{26,34,45,59}$ a pesar de ello, aún es una tarea de escasa difusión.

A pesar de que ya se ha identificado la dependencia del sistema de funciones ejecutivas para el desempeño en pruebas de fluidez verbal, no se ha precisado de forma suficiente esta relación. ${ }^{22}$ En la bibliografía se ha descrito que el componente de la fluidez que más se desarrolla en la infancia es el cambio (Switching) de una categoría a otra. Esta capacidad requiere de otras más básicas, como búsqueda estratégica, monitoreo y flexibilidad. También se ha descrito que la óptima comprensión de las oraciones pasivas (cuando no es claro que el sujeto es el agente de la acción descrita por el verbo) presupone el desarrollo de la capacidad de flexibilidad cognitiva, lo que permitiría ejercer un análisis de reversibilidad a los elementos lingüísticos expuestos. Esta capacidad empezaría a ser competente hasta los 6-7 años de edad. ${ }^{60}$

Desde la perspectiva clínica, se ha señalado la importancia de contar con instrumentos clínicos sensibles que identifiquen a los niños con trastorno de lenguaje. Por esta razón, las pruebas de fluidez de verbos podrían representar un campo de desarrollo clínico y cognitivo muy importante para la neuropsicología infantil. Pues permitirían, durante la niñez, caracterizar la organización semántica de los verbos, su tipo, forma, etc. ${ }^{61}$ No obstante que se reconoce a las tareas de fluidez de verbos como parte de las medidas clínicas en la evaluación del funcionamiento ejecutivo, es muy reciente su inclusión en baterías de evaluación de funciones ejecutivas. ${ }^{59} \mathrm{El}$ valor lineal del incremento del número de verbos que se produce en un minuto desde la infancia hasta la juventud, indica que esta medida de disponibilidad léxica puede ser un marcador importante del desarrollo neuropsicológico y psicolingüístico, ${ }^{47}$ tomando en cuenta que la competencia verbal-argumentativa máxima se alcanza hasta la adolescencia. ${ }^{19}$

\section{CONCLUSIONES}

A pesar de la relevancia que implica el uso del verbo en el desarrollo del lenguaje expresivo, las características lingüís- ticas y psicolingüísticas del desarrollo de la fluidez de verbos son campos insuficientemente explorados. ${ }^{8,60}$ Los estudios de caracterización durante el desarrollo normal permitirán conocer las particularidades básicas de este fenómeno y, posteriormente, permitirán identificar dificultades de forma temprana. Se destacaría así las dificultades específicas en el campo de la morfología y de la semántica verbal, lo que permitiría una mejor intervención-rehabilitación en las distintas alteraciones del neurodesarrollo que cursan con dificultades en el lenguaje. El control ejecutivo que subyace a la producción eficiente de verbos puede ser una medida sensible a dificultades "sutiles" (pero cognitivamente relevantes) de neurodesarrollo (enmascaradas o subclínicas) que no son detectadas por los métodos convencionales de evaluación.

\section{Financiamiento}

No aplica.

\section{Declaración de conflicto de intereses}

Los autores declararon no tener conflicto de intereses.

\section{REFERENCIAS}

1. Pinker S. Language learnability and language development. Cambridge: Cambridge University Press; 1984.

2. Álvarez A, Zinkgräf M, Casares MF, Olivares MA. Desarrollo del lenguaje en niños de 3 a 4 años: el paso a los procesos morfológicos. Revista Iberoamericana Educación 2005;35(1):1-10.

3. Scott RM, Fisher C. 2-year-olds use distributional cues to interpret transitivity alternating verbs. Lang Cogn Processes 2009;24(6):777-803.

4. Thordardottir ET, Weismer SE. High-frequency verbs and verb diversity in the spontaneous speech of school-age children with specific language impairment. Int J Lang Commun Disord 2001;36(2):221-244.

5. Murillo Rojas M. Diversidad de vocabulario en los preescolares. Aportes para valorar su competencia léxica. Filología Lingüística 2009;35(1):123-138.

6. Jia G, Kohnert K, Collado J, Aquino-Garcia F. Action naming in Spanish and English by sequential bilingual children and adolescents. J Speech Lang Hear Res 2006;49:588-602.

7. D'Amico S, Devescovi A, Bates E. Picture naming and lexical access in Italian children and adults. J Cogn Dev 2001;2(1):71-105.

8. Masterson J, Drukes J, Galiene D. Object and action picture naming in three and five year old children. J Child Lang 2008;35:373-402.

9. Parr N. Early verbs: a case of pragmatic bootstrapping? Working Papers Linguistics 2009;21:229-257.

10. Quillis A, Hernández Alonso C. Lingüística española aplicada a la terapia del lenguaje. España: Gredos; 1990.

11. Druks J, Masterson J. Object and Action Naming Battery. USA: Psychology Press; 2000.

12. Zelazo PD. The development of conscious control in childhood. Trends Cogn Sci 2004;8:12-17.

13. Baraff Bonawitz E, Ferranti D, Saxe R, Gopnick A et al. Just do it? Investigating the gap between prediction and action in toddlers' causal inference. Cognition 2010;115(1):104-117.

14. Vigliocco G, Vinson DP, Damian MF, Levelt W. Semantic distance effects on object and action naming. Cognition 2002;85:B61-B69.

15. Pye C, Loeb DF, Redmon S, Richardson LZ. When do children acquire verbs? En: Clark EV, (ed.). The proceedings of the twenty-sixth annual child language research forum. Stanford: Center for the Study of Language and Information; 1995.

16. Sánz Torrent M, Badia I, Serra M. Contributions from bilingual specific language impairment in Catalan and Spanish to the understan- 
ding of typical and pathological language acquisition. En: Pérez Vidal C, Bel Gaya A (eds.). A portait of the young in the new multilingual Spain. España: Multilingual Matters; 2008.

17. Kemmerer D, González Castillo J. The two-level theory of verb meaning: an approach to integrating the semantics of action with the mirror neuron system. Brain Lang 2010;112(1):54-76.

18. Aravena P, Hurtado E, Riveros R, Cardona JF, Manes F, Ibañez A. Applauding with Closed Hands: Neural Signature of Action-Sentence Compatibility Effects. PLoS ONE 2010;5(7):e11751.

19. Thordardottir E, Weismer S. Verb argument structure weakness in specific language impairment in relation to age and utterance lenght. Clin Linguist Phon 2002;16(4):233-250.

20. Holland SK, Plante E, Byars WB. Normal fMRI brain activation patterns in children performing a verb generation task. Neuroimage 2001;14:837-843.

21. Weiss EM, Siedentopf C, Hofer A, Deisenhammer EA. Brain activation patterns during a verbal fluency test in healthy male and female volunteers: a functional magnetic imaging study. Neurosci Lett 2003;352:191-194.

22. Spalek K, Thompson-Schill SL. Task-dependent semantic interference in language production: an fMRI study. Brain Lang 2008;107(3):220-228.

23. Vigliocco G, Vinson DP, Druks J, Barber $H$ et al. Nouns and verbs in the brain: a review of behavioural electrophysiological, neuropsychological and imaging studies. Neurosci Biobehav Rev 2011;35(3):407-426.

24. Dale P. Desarrollo del lenguaje: un enfoque psicolingüístico. México: Trillas; 1980.

25. Druks J. Verbs and nouns: A review of the literature. J Neurolinguist 2002;15:289-315.

26. Gainotti G, Silver MC, Daniele A, Gistolisi L. Neuroanatomical correlates of category-specific semantic disorders: a critical survey. Memory 1995;15:124-134.

27. Hillis AE, Tuffias E, Caramazza A. Modality-specific deterioration in naming verbs in nonfluent primary progressive aphasia. J Cogn Neurosci 2002;14(7):1.099-1.108.

28. Bedny M, Caramazza A. Perception, action and word meanings in the human brain: the case from action verbs. Ann N Y Acad Sci 2011;1224:81-95.

29. Kemmerer D, Gonzalez Castillo J, Talavage T, Patterson S et al. Neuroanatomical distribution of five semantic components of verbs: Evidence from fMRI. Brain Lang 2007;107:16-43.

30. Woods SP, Scott JC, Sires DA, Grant I et al. Action (verb) fluency: Test-retest reliability, normative standards, and construct validity. J Int Neuropsychol Soc 2005;11:408-415.

31. Szaflarski JP, Hoolland SK, Schmithorst VJ, Byars AW. An fMRI study of languaje lateralization in children and adults. Hum Brain Mapp 2006;27(3):202-212.

32. Bedny M, Caramazza A, Grossman E, Pascual-Leone A et al. Concepts are more than percepts: The case of action verbs. J Neurosci 2008;28:11347-11353.

33. Flores Lázaro JC, Ostrosky Shejet F. Desarrollo neuropsicológico de lóbulos frontales y funciones ejecutivas. México: Manual Moderno; 2012.

34. Piatt A, Fields J, Paolo AM, Troster AI. Action (verb naming) fluency as an executive function measure: convergent and divergent evidence of validity. Neuropsychologia 1999;37:1499-1503.

35. Shapiro L, Zurif E, Grimshaw J. Sentence processing and the mental representation of verbs. Cognition 1987;27:219-246.

36. Shapiro L, Zurif E, Grimshaw J. Verb processing during sentence comprehension: contextual impenetrability. J Psycholinguist Res 1989;18:223-243.

37. Shapiro L, Levine B. Verb processing during sentence comprehension in aphasia. Brain Lang 1990;38:21-47.

38. Lezak MD, Howieson DB, Loring DW. Neuropsychological assesment. New York: Oxford University Press; 2004.

39. Pang EW, Wang F, Malone M, Kadis Det al. Localization of Broca's area using verb generation tasks in the MEG: validation against fMRI. Neurosci Lett 2011;490:215-219.
40. Hirshorn EA, Thompson-Schill SL. Role of the left inferior frontal gyrus in covert word retrieval: neural correlates of switching during verbal fluency. Neuropsychologia 2006;44(12):2547-2557.

41. Marino J, Acosta Mesas A, Zorza JP. Control ejecutivo y fluidez verbal en población infantil: Medidas cuantitativas, cualitativas y temporales. Revista Psicología Ciencias Afines 2011;28(2):245-260.

42. Marino J, Díaz-Fajreldines H. Pruebas de fluidez verbal categoriales, fonológicas y gramaticales en la infancia: factores ejecutivos y semánticos. Revista Chilena Neuropsicología 2011;6(1):48-55.

43. Bjorklund DF, Dukes C, Douglas Brown R. The development of memory strategies. In: Courage M, Cowan N, editors, The development of memory in infant and childhood. New York: Psychology Press; 2009; p.145-175.

44. Miller PH. How to best utilize a deficiency. Child Dev 2000;71(4):10131017.

45. Flores Lázaro JC, Tinajero Carrasco B, Castro Ruiz B. Influencia del nivel y de la actividad escolar en las funciones ejecutivas. Rev Interam Psicol 2011;45(2):281-292.

46. Ávila R. Lo que hacen los niños: verbos y grado escolar. Studia Romanica Posnaniensia. 2004;31:129-146.

47. Flores Lázaro JC, Ostrosky-Solís F. Developmental characteristics in category generation reflects different prefrontal cortex maturation. Advances Psychology Research 2008;55:3-13.

48. Sheng L, McGregor KK. Object and action naming in children with specific language impairment. J Speech Lang Hear Res 2010;53(6):1704-1719.

49. Den Ouden DB, Fix S, Parrish TB, Thompson CK. Argument structure effects in action verb naming in static and dynamic conditions. J Neurolinguistics 2009;22(2):196-215.

50. Baron IS, Erickson K, Ahronovich MD, Coulehan K et al. Visuospatial and verbal fluency relative deficits in 'complicated' late-preterm preschool children. Early Hum Dev 2009;85(12):751-754.

51. Karunanaya P, Schmithorst VJ, Vannest J, Szaflarski JP et al. A linear structural equation model for covert verb generation based on independent component analysis of fMRI data from children and adolescents. Front Syst Neurosci 2011;5:29.

52. Dubé S, Le Normand MT, Cohen H. Acquisition of lexical morphology in simple partial epilepsy. Brain Lang 2001;78(1):109-114.

53. Marini A, Lorusso ML, D'Angelo MG, Civati $F$ et al. Evaluation of narrative abilities in patients suffering from Duchenne Muscular Dystrophy. Brain Lang 2007;102(1):1-12.

54. Wagner CR, Nettelbladt U, Sahlén B, Nilholm C. Conversation versus narration in pre-school children with language impairment. Int J Lang Commun Disord 2000;35(1):83-93.

55. Rice M, Bode J. GAPS in the verb lexicons of children with specific language impairment. First Lang 1993;13:113-131.

56. Bedore LM, Leonard LB. Verb inflections and noun phrase morphology in the spontaneous speech of Spanish-speaking children whit specific language impairment. Appl Psycholinguist 2005;26(2):195-225.

57. Sanz Torrent M. Los verbos en niños con trastorno de lenguaje. Rev Logop Foniatr Audiol 2002;22:100-110.

58. Pérez Morales MF, Bittencourt Chastinet J, Flores Lázaro JC. Daño prefrontal al inicio de la adolescencia: Comparación de dos casos. Revista Neuropsicología, Neuropsiquiatría Neurociencias 2011;11(2):35-48.

59. Flores Lázaro JC, Ostrosky-Solís F, Lozano Gutiérrez A. Batería de funciones frontales y ejecutivas: presentación. Revista Neuropsicología, Neuropsiquiatría Neurociencias 2008;8(1):141-158.

60. Álvarez A, Casares MF, Zinkgräf M. Construcciones pasivas en español argentino como lengua materna: un estudio de comprensión en niños de 3 años. Revista Iberoamericana Educación 2008:46(6):1-9.

61. Jackson-Maldonado D. La identificación del trastorno específico de lenguaje en niño hispano-hablantes por medio de pruebas formales e informales. Revista Neuropsicología, Neuropsiquiatría Neurociencias. 2011;11(1):33-50. 